\title{
Egresos hospitalarios por enfermedades otorrinolaringológicas, Chile 2018
}

\author{
Hospital discharges for otorhinolaryngological diseases, Chile 2018 \\ Esteban Ortúzar G. ${ }^{1,2}$, Jorge Godoy A. ${ }^{1,2}$, Domingo Lancellotti G. ${ }^{2}$, Carlos Namoncura P. ${ }^{1,2}$
}

\section{Resumen}

Introducción: Las enfermedades otorrinolaringológicas presentan una alta prevalencia en la población, lo que obliga continuamente a estudiar sus características epidemiológicas. Una forma de estimarlo a nivel país es a través de los egresos hospitalarios. Objetivo: Describir las principales causas de egresos hospitalarios por enfermedades otorrinolaringológicas a nivel nacional durante el año 2018. Material y Método: Estudio transversal donde se describen las distintas frecuencias de las principales causas de egresos hospitalarios por causa otorrinolaringológica en todo Chile durante el año 2018. Datos obtenidos del Departamento de Estadísticas e Información en Salud (DEIS). Resultados: Se incluyeron 64.715 egresos con una mediana de edad de 22 años. Las patologías con mayor cantidad de egresos fueron la enfermedad crónica de amígdalas y adenoides $(28,4 \%)$, patología rinosinusal $(23,3 \%)$, neoplasias de cabeza y cuello $(15,2 \%)$ y oído $(7,8 \%)$. Conclusión: La enfermedad crónica de amígdalas y adenoides persiste como la patología con más egresos. Emergen en importancia enfermedades neoplásicas y diagnósticos relacionados con mejora de calidad de vida. Existen errores de codificación de diagnósticos que requieren discusión dentro de la especialidad para mejorar la calidad del registro.

Palabras clave: Egresos hospitalarios, enfermedades otorrinolaringológicas, prevalencia.

\begin{abstract}
Introduction: Otolaryngological diseases have a high prevalence in the population, creating the need to continuously study their epidemiological characteristics. One way to estimate prevalence at the country level, is to check hospital discharges. Aim: To describe the main causes of hospital discharges due to otolaryngological diseases at the Chilean level during 2018. Material and Method: Cross-sectional study describing the different frequencies of the main causes of hospital discharge due to otolaryngological diseases throughout Chile during 2018. Data obtained from the Department of Health Statistics and Information (DEIS). Results: 64,715 discharges were included with a median age of 22 years. The pathologies with the highest number of discharges were chronic tonsil and adenoids Disease (28.4\%), rhinosinusal (23.3\%), head and neck neoplasms (15.2\%) and ear (7.8\%). Conclusion: Chronic tonsil and adenoids disease persists as the pathology with the most discharges. Neoplastic diseases and diagnoses related to improvement of quality of life emerge in importance. There are errors in the coding of diagnoses that require discussion within the specialty to improve the quality of the registry.
\end{abstract}

Keywords: Hospital discharges, otolaryngological diseases, prevalence.

\author{
'Servicio de \\ Otorrinolaringología, Hospital \\ San Juan de Dios. La Serena, \\ Chile. \\ Universidad Católica del \\ Norte. Coquimbo, Chile. \\ Los autores declaran no tener \\ conflictos de interés. \\ Recibido el 29 de marzo de \\ 2021. Aceptado el 20 de \\ agosto de 2021. \\ Correspondencia: \\ Esteban Ortúzar G. \\ Juan Cisternas 1960, oficina \\ 23. La Serena, Chile. \\ Email: esteban.ortuzar@ucn.cl
}

\section{Introducción}

Las enfermedades otorrinolaringológicas son un grupo de patologías de importancia considerable, dada su alta frecuencia y transversalidad etaria en que se presentan ${ }^{1,2}$. Son responsables de ausentismo laboral y escolar, producen alteración de la calidad de vida y se asocian a mortalidad en poblaciones extremas ${ }^{3}$. Esto obliga a estimar continuamente su incidencia o prevalencia con fines de planificación en salud, lo cual a nivel "macro país" es com- 
plejo de conocer. Una forma de cuantificarlo es mediante los egresos hospitalarios de causa otorrinolaringológica que, si bien corresponden a un subconjunto de la morbilidad y son condicionados por su complejidad e infraestructura disponible, nos pueden dar una estimación de las características y frecuencias de las distintas enfermedades relacionadas con la especialidad 4 .

No existen publicaciones actualizadas sobre egresos hospitalarios de causa otorrinolaringológica a nivel chileno. El último estudio fue realizado con estadísticas del año 1985 en donde se describieron 34.492 egresos con un $65 \%$ de población infantil y donde destacó como principal diagnóstico la patología crónica de amígdalas (38\% de los egresos), seguido de patología de oído $(10 \%)^{5}$. Los reportes posteriores han sido sólo de centros individuales: Ulloa y cols. describieron una casuística de 5 años (1993-1997) donde analizaron un total de 8.725 egresos del Servicio de Otorrinolaringología del Hospital de Concepción. Los pacientes menores de 20 años concentraron el $50 \%$ de los egresos y la patología faríngea fue la principal enfermedad diagnosticada ${ }^{6}$. Más recientemente, Domínguez y cols. del Hospital Clínico de la Universidad de Chile, publicaron un estudio que reunió información de egresos entre los años 2007 y 2014, donde destacó la patología crónica de amígdalas como primera causa de egreso, seguido de enfermedades de oído y nasosinusales ${ }^{7}$.

En Chile existe el sistema de registro de grupos relacionados por el diagnóstico (GRD) que permite tener a nivel nacional una estadística de egresos hospitalarios, tanto público como privado ${ }^{8}$. Este sistema entrega un registro sistemático de datos demográficos y diagnósticos con clasificación CIE-10, lo que lo transforma en una herramienta útil para fines de investigación.

\section{Objetivo}

Este estudio tiene como objetivo general describir las principales causas de egresos hospitalarios por enfermedades otorrinolaringológicas a nivel chileno durante el año 2018. Los objetivos específicos plantean realizar una descripción demográfica general y de los distintos grupos de enfermedades, mostrar los principales diagnósticos a nivel nacional y regional, y en el sistema público y privado. Además, es de interés obtener información relevante para fines de gestión en salud.

\section{Material y Método}

Se realizó un estudio de corte transversal donde se describen los egresos hospitalarios por causa otorrinolaringológica a nivel país, año 2018. Los datos fueron obtenidos del Departamento de Estadísticas e Información en Salud (DEIS) considerando instituciones públicas y privadas ${ }^{9}$. Se incluyeron todos los diagnósticos relacionados con la especialidad según clasificación CIE-10, independiente del servicio o especialidad que realiza el egreso. Esto contempla pacientes que requirieron hospitalización para manejo médico de su patología, cirugía mayor hospitalizada y cirugía menor ambulatoria. Los procedimientos ambulatorios no están incluidos en la base de datos del DEIS. Se excluyen patologías traumáticas de cabeza y cuello de manejo habitual por maxilofacial, neurocirugía y cirugía general. El procesamiento de la información fue mediante estadística descriptiva e inferencial, utilizando la prueba de chi-cuadrado. Las diferencias fueron establecidas como significativas a una $\mathrm{p}<0,05$. Las tasas de egresos fueron calculadas por 100.000 habitantes en base a la población estimada para el mismo año según censo del año $2017^{10}$. Los análisis fueron realizados mediante Microsoft Excel y SPSS en su versión 25.

\section{Resultados}

Durante el año 2018 hubo un total de 1.048.576 egresos hospitalarios, de los cuales $64.715(6,8 \%)$ fueron por causa otorrinolaringológica. La mediana de días hospitalizados fue de un día, con un rango intercuartil (R.I) de 1-2. El 57,5\% de los egresos fueron del sector privado y un $68,4 \%$ del total correspondió a pacientes intervenidos quirúrgicamente. El 51\% fueron del sexo masculino, con una mediana de edad de 22 años (R.I = 6-44). Se observó un predominio de población adulta que alcanzó el 50,5\%, seguido de pacientes 
menores de 15 años y adultos mayores $(\geq 65$ años) con un $40,9 \%$ y $8,6 \%$ respectivamente (Figura 1).

En la Tabla 1 se muestran los principales 25 diagnósticos individuales que corresponden al $80,1 \%$ del total de egresos. El diagnóstico más frecuente fue enfermedad crónica de las amígdalas y adenoides, con un $28,4 \%$ del total seguido por desviación del tabique nasal, tumor maligno del tiroides, absceso periamigdalino y rinosinusitis crónica con porcentajes entre $12,9 \%$ y $3,3 \%$. Otros diagnósticos de interés fueron otitis media no especificada $\left(7^{\circ}\right)$, patologías infecciosas en población infantil como "laringitis aguda" $\left(10^{\circ}\right)$, "rinofaringitis aguda" $\left(12^{\circ}\right)$ y patología neoplásica como "tumor maligno de la piel" $\left(11^{\circ}\right)$, "tumor maligno de cavidad oral" $\left(16^{\circ}\right)$ y "tumor maligno de laringe" $\left(22^{\circ}\right)$.

En la Tabla 2 se describen las patologías agrupadas. La enfermedad crónica de amígdalas y adenoides siguió siendo la de mayor frecuencia $(28,4 \%)$, secundada por patología rinosinusal que reunió al 23,4\% de los egresos. Les sigue las patologías neoplásicas de cabeza y cuello, con un $15,3 \%$ del total, las enfermedades de oído, con un 7,8\%, y las patologías cócleo-vestibular con un 2,6\%. El porcentaje restante se agrupó a nuestro criterio en un grupo que denominamos "otras patologías benignas de vía aerodigestiva superior", que reúne una gran cantidad de diagnósticos heterogéneos entre sí incluyendo enfermedades crónicas, congénitas e infecciosas.

La región con mayor cantidad de egresos fue la Región Metropolitana, abarcando el $46,7 \%$ del total nacional, secundada por la Región del Bío-Bío (12,8\%) y la Región de Valparaíso (7,7\%). Al comparar las tasas de egresos destaca en primer lugar la Región de Aysén con 539 egresos por 100.000 habs., seguida por la Región del Bío-Bío y Región de Magallanes con 503 y 414 por 100.000 habs., respectivamente (Tabla 3 ).

Como recién se describió, la enfermedad crónica de amígdalas y adenoides es la principal causa de egreso $(28,4 \% ; n=18.396)$. El 66,7\% correspondió a egresos del sector privado, con una mediana de días de hospitalización de 1 $($ R.I. $=1-1)($ Tabla 4$)$. Un 53\% de los pacientes fue de sexo masculino, con un predominio de la población infantil que alcanzó el 84,0\% de

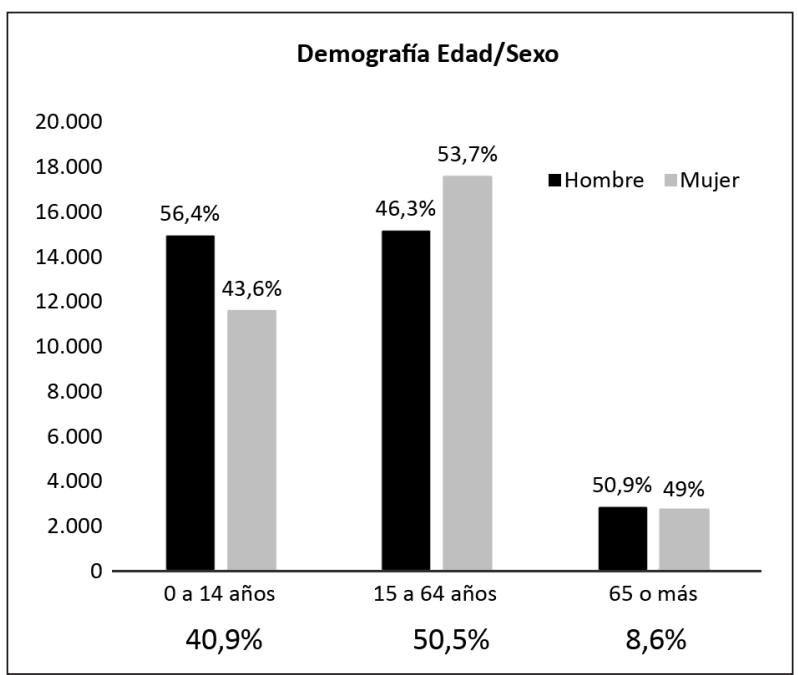

Figura 1. Distribución etaria de los diagnósticos otorrinolaringológicos de los egresos hospitalarios, Chile, año 2018.

pacientes menores de 15 años con una mediana de edad de 6 años (R.I. = 4-10) (Tabla 5).

La región con mayor número de egresos por enfermedad crónica de amígdalas y adenoides fue la Región Metropolitana (34,7\%) seguida de la Región del Bío-Bío (13,5\%) y Región de Valparaíso (7,9\%). Sin embargo, en términos de tasas aparece en primer lugar la Región de Aysén (190 por 100.000 habs.), secundada por la Región del Nuble y Región del Bío-Bío (Tabla 6).

La patología rinosinusal fue responsable del $23,3 \%$ de los egresos $(n=15.118)$. El 74,6\% de estos provienen de instituciones privadas con una mediana de días de hospitalización de 1 $($ R.I. $=1-1)$, y un $83,1 \%$ de pacientes intervenidos quirúrgicamente. El 54\% corresponde al sexo masculino, con un predominio de pacientes adultos $(85,7 \%)$ y una mediana de edad de 32 años (RI = 22-46) (Tablas 4 y 5$)$. El diagnóstico más frecuente fue desviación del tabique nasal con un 55,5\% seguido de sinusitis crónica $(14,0 \%)$, fractura de los huesos de la nariz $(11,7 \%)$, sinusitis aguda $(3,9 \%)$, y epistaxis e hipertrofia de cornetes con un 3,3\% y $3 \%$ respectivamente (Tabla 7 ). La región con mayor proporción de egresos fue la Región Metropolitana, sin embargo, la Región de Magallanes emerge como la región con mayor tasa de egreso (Tabla 6). 


\section{Tabla 1. Principales diagnósticos de egresos otorrinolaringológicos}

\begin{tabular}{|c|c|c|c|c|c|}
\hline Diagnósticos individuales & $\begin{array}{l}\text { Código (s) } \\
\text { CIE-10 }\end{array}$ & $\mathbf{n}$ & $\begin{array}{l}\% \text { del total } \\
\text { de egresos }\end{array}$ & $\begin{array}{l}\text { \% de egresos } \\
\text { públicos }\end{array}$ & $\begin{array}{c}\% \text { de egresos } \\
\text { privados }\end{array}$ \\
\hline Enfermedad crónica de las amígdalas y adenoides & J35.X & 18.396 & $28,4 \%$ & $33,3 \%$ & $66,7 \%$ \\
\hline Desviación de tabique nasal & J34.2 & 8.322 & $12,9 \%$ & $16,8 \%$ & $83,2 \%$ \\
\hline Tumor maligno de glándula tiroides & C73.X & 4.437 & $6,9 \%$ & $45 \%$ & $55 \%$ \\
\hline Absceso periamigdalino & J36.X & 3.137 & $4,8 \%$ & $58,7 \%$ & $41,3 \%$ \\
\hline Rinosinusitis crónica (con y sin pólipos) & $\begin{array}{l}\text { J32.X; } \\
\text { J33.X }\end{array}$ & 2.115 & $3,3 \%$ & $37,6 \%$ & $62,4 \%$ \\
\hline Fractura de los huesos de la nariz & S02.2 & 1.769 & $2,7 \%$ & $30 \%$ & $70 \%$ \\
\hline Otitis media no especificada & H66.9 & 1.501 & $2,3 \%$ & $75 \%$ & $25 \%$ \\
\hline Amigdalitis aguda & J03.X & 1.418 & $2,2 \%$ & $50,4 \%$ & $49,6 \%$ \\
\hline Fisura labiopalatina & $\begin{array}{l}\text { Q35.X; } \\
\text { Q36.X; } \\
\text { Q37.X }\end{array}$ & 1.327 & $2,1 \%$ & $67,4 \%$ & $32,6 \%$ \\
\hline Laringitis aguda & $\begin{array}{l}\text { J04.0; } \\
\text { J05.X }\end{array}$ & 1.174 & $1,8 \%$ & $71,7 \%$ & $28,3 \%$ \\
\hline Tumor maligno de la piel & $\begin{array}{l}\text { C44.0; } \\
\text { C44.1; } \\
\text { C44.2; } \\
\text { C44.3; } \\
\text { C44.4 }\end{array}$ & 1.128 & $1,7 \%$ & $45,3 \%$ & $54,7 \%$ \\
\hline Rinofaringitis aguda & J00.X & 818 & $1,3 \%$ & $77 \%$ & $23 \%$ \\
\hline Vértigo periférico & $\begin{array}{l}\text { H81.0; } \\
\text { H81.1; } \\
\text { H81.2; } \\
\text { H81.3 }\end{array}$ & 774 & $1,2 \%$ & $47,4 \%$ & $52,6 \%$ \\
\hline Sinusitis aguda & J01.X & 584 & $0,9 \%$ & $35,3 \%$ & $64,7 \%$ \\
\hline Celulitis o absceso de piso de boca & $\mathrm{K} 12.2$ & 555 & $0,9 \%$ & 75,9 & 24,1 \\
\hline Tumor maligno de cavidad oral & $\begin{array}{l}\text { C00.X; } \\
\text { C02.X*; } \\
\text { C03.X; } \\
\text { C04.X; } \\
\text { C05.X; } \\
\text { C06.X }\end{array}$ & 525 & $0,8 \%$ & $68,8 \%$ & $31,2 \%$ \\
\hline Epistaxis & R04.0 & 497 & $0,8 \%$ & $67,6 \%$ & $32,4 \%$ \\
\hline Vértigo no especificado & $\begin{array}{l}\text { H81.8; } \\
\text { H81.9; } \\
\text { H82.X }\end{array}$ & 491 & $0,8 \%$ & $56,5 \%$ & $43,4 \%$ \\
\hline Otitis media por efusión & $\begin{array}{l}\text { H65.2; } \\
\text { H65.3 }\end{array}$ & 485 & $0,7 \%$ & $25,2 \%$ & $74,8 \%$ \\
\hline Hipertrofia de cornetes & J34.3 & 454 & $0,7 \%$ & $18,3 \%$ & $81,7 \%$ \\
\hline Colesteatoma del oído medio & $\mathrm{H} 71 . \mathrm{X}$ & 448 & $0,7 \%$ & $61,4 \%$ & $38,6 \%$ \\
\hline Tumor maligno de laringe & C32.X & 402 & $0,6 \%$ & $79,6 \%$ & $20,4 \%$ \\
\hline Anquiloglosia & Q38.1 & 368 & $0,6 \%$ & $56,3 \%$ & $43,7 \%$ \\
\hline Tumor benigno de glándulas salivales & D11.X & 339 & $0,5 \%$ & $35 \%$ & $65 \%$ \\
\hline Pólipo de cuerda vocal & J38.1 & 331 & $0,5 \%$ & $25 \%$ & $75 \%$ \\
\hline Otros & - & 12.930 & $19,9 \%$ & - & - \\
\hline Total & - & 64.715 & $100 \%$ & - & - \\
\hline
\end{tabular}


Tabla 2. Diagnósticos ORL agrupados

\begin{tabular}{lcc}
\hline Diagnósticos agrupados & $\mathbf{n}$ & \% del total de egresos \\
\hline Enfermedad crónica de amígdalas y adenoides & 18.396 & $28,4 \%$ \\
\hline Patología rinosinusal & 15.118 & $23,4 \%$ \\
\hline Patología neoplásica & 9.887 & $15,3 \%$ \\
\hline Patología de oído & 5.032 & $7,8 \%$ \\
\hline Patología cócleo-vestibular & 1.705 & $2,6 \%$ \\
Otras patologías benignas de VADS & 14.577 & $22,5 \%$ \\
\hline Total & 64.715 & $100 \%$ \\
\hline
\end{tabular}

Tabla 3. Egresos ORL general. Porcentaje por región. Tasa x 100.000 habitantes

\begin{tabular}{cccc}
\hline Región & \multicolumn{1}{c}{$\mathbf{n}$} & \% Bruto de egresos hospitalarios & Tasa $\mathbf{x} 100.000$ \\
XV & 309 & $0,5 \%$ & 127,7 \\
I & 596 & $0,9 \%$ & 167,9 \\
II & 2.322 & $3,6 \%$ & 360,0 \\
III & 791 & $1,2 \%$ & 257,0 \\
IV & 1.727 & $2,7 \%$ & 213,9 \\
V & 4.948 & $7,7 \%$ & 259,0 \\
XIII & 30.181 & $46,7 \%$ & 391,8 \\
VI & 2.315 & $3,6 \%$ & 239,5 \\
VII & 2.384 & $3,7 \%$ & 215,6 \\
XVI & 1.774 & $2,7 \%$ & 351,8 \\
VIII & 8.288 & $12,8 \%$ & 503,7 \\
IX & 2.963 & $4,6 \%$ & 295,9 \\
XIV & 1.520 & $2,4 \%$ & 379,1 \\
X & 3.254 & $5,0 \%$ & 370,9 \\
XI & 572 & $0,9 \%$ & 539,5 \\
XII & 720 & $1,1 \%$ & 414,8 \\
\hline Total, País & 64.715 & $100 \%$ & 345,1 \\
\hline
\end{tabular}

Tabla 4. Egresos hospitalarios ORL según sistema de salud y cirugía

\begin{tabular}{|c|c|c|c|c|c|c|c|c|c|c|}
\hline \multirow[b]{2}{*}{ Patología } & \multicolumn{2}{|c|}{ Egresos } & \multicolumn{4}{|c|}{ Días hospitalización } & \multicolumn{2}{|c|}{ Cirugía } & \multicolumn{2}{|c|}{ Condición de egreso } \\
\hline & Público & Privado & Mediana & R.I. & Promedio & D.E. & Sí & No & Vivo & Fallecido \\
\hline $\begin{array}{l}\text { Enfermedad crónica } \\
\text { de amígdalas }\end{array}$ & $33,3 \%$ & $66,7 \%$ & 1 & $1-1$ & 1,2 & 0,8 & $93,5 \%$ & $6,5 \%$ & $99,9 \%$ & $0,1 \%$ \\
\hline Rinosinusal & $25,4 \%$ & $74,6 \%$ & 1 & $1-1$ & 1,65 & 2,9 & $83,1 \%$ & $16,9 \%$ & $99,9 \%$ & $0,1 \%$ \\
\hline Neoplasias & $53,2 \%$ & $46,8 \%$ & 2 & $1-4$ & 4,9 & 10,8 & $66,6 \%$ & $33,4 \%$ & $98,4 \%$ & $1,6 \%$ \\
\hline Oído & $53,7 \%$ & $46,3 \%$ & 1 & $1-2$ & 2,1 & 4,3 & $76,1 \%$ & $23,9 \%$ & $100 \%$ & $0 \%$ \\
\hline Cócleo-vestibular & $52 \%$ & $48 \%$ & 2 & $1-4$ & 3,4 & 4,1 & $14,5 \%$ & $85,5 \%$ & $99,9 \%$ & $0,1 \%$ \\
\hline VADS & $60,6 \%$ & $37,4 \%$ & 2 & $1-4$ & 3,4 & 6,9 & $27,5 \%$ & $72,5 \%$ & $99,6 \%$ & $0,4 \%$ \\
\hline Total & $42,5 \%$ & $57,5 \%$ & 1 & $1-2$ & 2,5 & 9,6 & $68,4 \%$ & $31,6 \%$ & $99,6 \%$ & $0,36 \%$ \\
\hline
\end{tabular}


Tabla 5. Egresos hospitalarios ORL según género y edad

\begin{tabular}{|c|c|c|c|c|c|c|c|c|c|c|c|}
\hline \multirow[b]{2}{*}{ Patología } & \multicolumn{3}{|c|}{ Género } & \multicolumn{4}{|c|}{ Grupos edad } & \multicolumn{4}{|c|}{ Edad (años) } \\
\hline & Hombre & Mujer & $P$ value & $<15$ & $15-64$ & $>64$ & $P$ value & Mediana & R.I. & Promedio & D.E. \\
\hline $\begin{array}{l}\text { Enfermedad } \\
\text { crónica de } \\
\text { amígdalas }\end{array}$ & $53 \%$ & $47 \%$ & $<0,01$ & $84 \%$ & $15,9 \%$ & $0,1 \%$ & $<0,01$ & 6 & $4-10$ & 9,4 & 9,5 \\
\hline Rinosinusal & $54 \%$ & $46 \%$ & $<0,01$ & $8,4 \%$ & $85,7 \%$ & $5,9 \%$ & $<0,01$ & 32 & $22-46$ & 34,5 & 17,2 \\
\hline Neoplasia & $39 \%$ & $61 \%$ & $<0,01$ & $1,8 \%$ & $67,7 \%$ & $30,6 \%$ & $<0,01$ & 55 & $41-68$ & 53,7 & 18,4 \\
\hline Oído & $50 \%$ & $50 \%$ & 0,55 & $45,1 \%$ & $49 \%$ & $5,9 \%$ & $<0,01$ & 18 & $7-47$ & 26,8 & 22,4 \\
\hline Cócleo vestibular & $45 \%$ & $55 \%$ & $<0,01$ & $14 \%$ & $55,7 \%$ & $30,3 \%$ & $<0,01$ & 55 & $36-76$ & 50,0 & 23,8 \\
\hline VADS & $53 \%$ & $47 \%$ & $<0,01$ & $48,4 \%$ & $46 \%$ & $5,6 \%$ & $<0,01$ & 16 & $2-34$ & 21,3 & 21,9 \\
\hline Total Chile & $51 \%$ & $49 \%$ & $<0,01$ & $40,9 \%$ & $50,5 \%$ & $8,6 \%$ & $<0,01$ & 22 & $6-4$ & 27,2 & 23,2 \\
\hline
\end{tabular}

\begin{tabular}{|c|c|c|c|c|c|c|c|c|c|c|c|c|}
\hline \multirow[b]{2}{*}{ Región } & \multicolumn{2}{|c|}{$\begin{array}{l}\text { Enfermedad } \\
\text { crónica de las } \\
\text { amígdalas }\end{array}$} & \multicolumn{2}{|c|}{$\begin{array}{l}\text { Patología } \\
\text { rinosinusal }\end{array}$} & \multicolumn{2}{|c|}{$\begin{array}{l}\text { Patología } \\
\text { neoplásica }\end{array}$} & \multicolumn{2}{|c|}{$\begin{array}{l}\text { Patología de } \\
\text { oído }\end{array}$} & \multicolumn{2}{|c|}{$\begin{array}{l}\text { Patología } \\
\text { cócleo- } \\
\text { vestibular }\end{array}$} & \multicolumn{2}{|c|}{$\begin{array}{l}\text { Patología } \\
\text { benigna de } \\
\text { VADS }\end{array}$} \\
\hline & $\begin{array}{l}\% \text { Bruto } \\
\text { del total } \\
\text { nacional }\end{array}$ & $\begin{array}{c}\text { Tasa x } \\
100.000 \\
\text { habs. }\end{array}$ & $\begin{array}{l}\% \text { Bruto } \\
\text { del total } \\
\text { nacional }\end{array}$ & $\begin{array}{c}\text { Tasa x } \\
100.000 \\
\text { habs. }\end{array}$ & $\begin{array}{l}\% \text { Bruto } \\
\text { del total } \\
\text { nacional }\end{array}$ & $\begin{array}{c}\text { Tasa x } \\
100.000 \\
\text { habs. }\end{array}$ & $\begin{array}{l}\% \text { Bruto } \\
\text { del total } \\
\text { nacional }\end{array}$ & $\begin{array}{c}\text { Tasa x } \\
100.000 \\
\text { habs. }\end{array}$ & $\begin{array}{l}\% \text { Bruto } \\
\text { del total } \\
\text { nacional }\end{array}$ & $\begin{array}{c}\text { Tasa x } \\
100.000 \\
\text { habs. }\end{array}$ & $\begin{array}{l}\% \text { Bruto } \\
\text { del total } \\
\text { nacional }\end{array}$ & $\begin{array}{c}\text { Tasa } x \\
100.000 \\
\text { habs. }\end{array}$ \\
\hline$X V$ & $0,1 \%$ & 8,3 & $0,42 \%$ & 26,0 & $0,6 \%$ & 25,6 & $0,6 \%$ & 12,0 & $1,3 \%$ & 9,1 & $0,8 \%$ & 46,7 \\
\hline I & $0,7 \%$ & 38,6 & $0,66 \%$ & 28,2 & $1,4 \%$ & 37,8 & $0,9 \%$ & 12,4 & $1,0 \%$ & 4,8 & $1,1 \%$ & 46,2 \\
\hline$\|$ & $4,8 \%$ & 137,0 & $3,32 \%$ & 77,8 & $3,5 \%$ & 54,0 & $2,6 \%$ & 20,2 & $2,2 \%$ & 5,9 & $2,9 \%$ & 65,3 \\
\hline III & $1,7 \%$ & 100,4 & $0,85 \%$ & 41,9 & $0,7 \%$ & 24,0 & $2,2 \%$ & 36,4 & $1,6 \%$ & 8,8 & $1,0 \%$ & 45,5 \\
\hline IV & $3,9 \%$ & 88,3 & $2,59 \%$ & 48,4 & $1,9 \%$ & 23,4 & $2,5 \%$ & 15,5 & $1,2 \%$ & 2,5 & $2,0 \%$ & 35,8 \\
\hline V & $7,9 \%$ & 76,5 & $6,87 \%$ & 54,3 & $7,9 \%$ & 41,0 & $5,8 \%$ & 15,4 & $6,6 \%$ & 5,9 & $8,7 \%$ & 66,5 \\
\hline XIII (RM) & $34,7 \%$ & 82,9 & $53,86 \%$ & 105,6 & $58,0 \%$ & 74,4 & $49,6 \%$ & 32,4 & $46,6 \%$ & 10,3 & $45,6 \%$ & 86,2 \\
\hline VI & $5,5 \%$ & 104,6 & $3,06 \%$ & 47,8 & $1,4 \%$ & 14,1 & $3,0 \%$ & 15,7 & $1,8 \%$ & 3,2 & $3,6 \%$ & 54,1 \\
\hline VII & $5,1 \%$ & 84,9 & $2,28 \%$ & 31,1 & $2,9 \%$ & 26,0 & $3,5 \%$ & 15,8 & $3,2 \%$ & 5,0 & $4,0 \%$ & 52,8 \\
\hline $\mathrm{XVI}$ & $4,7 \%$ & 170,9 & $2,19 \%$ & 65,6 & $1,0 \%$ & 19,2 & $2,9 \%$ & 28,6 & $2,5 \%$ & 8,3 & $2,0 \%$ & 59,1 \\
\hline VIII & $13,5 \%$ & 150,7 & $13,73 \%$ & 126,0 & $11,6 \%$ & 69,5 & $14,6 \%$ & 44,6 & $14,7 \%$ & 15,2 & $11,0 \%$ & 97,6 \\
\hline IX & $5,6 \%$ & 102,4 & $3,28 \%$ & 49,5 & $3,4 \%$ & 33,3 & $3,5 \%$ & 17,5 & $4,9 \%$ & 8,3 & $5,8 \%$ & 85,0 \\
\hline XIV & $2,4 \%$ & 108,2 & $1,70 \%$ & 64,1 & $2,4 \%$ & 59,4 & $1,4 \%$ & 17,0 & $3,2 \%$ & 13,5 & $3,2 \%$ & 117,0 \\
\hline$x$ & $7,2 \%$ & 151,8 & $3,01 \%$ & 51,7 & $2,4 \%$ & 27,5 & $4,4 \%$ & 25,4 & $5,8 \%$ & 11,2 & $6,2 \%$ & 103,3 \\
\hline XI & $1,1 \%$ & 190,5 & $0,55 \%$ & 78,3 & $0,3 \%$ & 25,5 & $1,6 \%$ & 74,5 & $0,6 \%$ & 9,4 & $1,2 \%$ & 161,3 \\
\hline XII & $1,0 \%$ & 105,4 & $1,62 \%$ & 141,2 & $0,6 \%$ & 35,1 & $1,1 \%$ & 30,5 & $2,9 \%$ & 28,8 & $0,9 \%$ & 73,7 \\
\hline
\end{tabular}




\section{Tabla 7. Principales diagnósticos ORL por grupos}

\begin{tabular}{|c|c|c|c|}
\hline & CIE-10 & $\mathbf{n}$ & $\%$ \\
\hline \multicolumn{4}{|l|}{ Enfermedad crónica de las amígdalas y adenoides } \\
\hline Amigdalitis crónica & J35.0 & 2.630 & $14,3 \%$ \\
\hline Hipertrofia de las amígdalas & J35.1 & 2.264 & $12,3 \%$ \\
\hline Hipertrofia de los adenoides & J35.2 & 2.309 & $12,5 \%$ \\
\hline Hipertrofia de las amígdalas y adenoides & J35.3 & 10.407 & $56,6 \%$ \\
\hline Otros amígdalas y adenoides & J35.8; J35.9 & 786 & $4,3 \%$ \\
\hline Total & & 18.396 & $100 \%$ \\
\hline \multicolumn{4}{|l|}{ Rinosinusal } \\
\hline Desviación del tabique nasal & $\mathrm{J} 34.2$ & 8.392 & $55,5 \%$ \\
\hline Rinosinusitis crónica (con y sin pólipos) & J32.X; J33.X & 2.115 & $14 \%$ \\
\hline Fractura nasal & S02.2 & 1.769 & $11,7 \%$ \\
\hline Sinusitis aguda & J01.X & 584 & $3,9 \%$ \\
\hline Epistaxis & R04.0 & 497 & $3,3 \%$ \\
\hline Hipertrofia de cornetes & J34.3 & 454 & $3 \%$ \\
\hline Rinitis, cuerpo extraño nasal, otros & J30.X; S01.2; T17.1 & 1.307 & $8,6 \%$ \\
\hline Total & & 15.118 & $100 \%$ \\
\hline \multicolumn{4}{|l|}{ Neoplasias malignas* } \\
\hline Tumor maligno del tiroides & C73.X & 4.437 & $58,3 \%$ \\
\hline Tumor maligno de piel de cabeza y cuello & $\begin{array}{l}\text { C44.0; C44.1; C44.2; } \\
\text { C44.3; C44.4 }\end{array}$ & 1.128 & $14,8 \%$ \\
\hline Tumor maligno de cavidad oral & $\begin{array}{l}\text { C00.X; C02.X; C03.X; } \\
\text { C04.X; C05.X; C06.X }\end{array}$ & 525 & $6,9 \%$ \\
\hline Tumor maligno de laringe & C32.X & 402 & $5,3 \%$ \\
\hline Tumor maligno de orofaringe & C10.X; C09.X; C01.X; C02.4 & 297 & $3,9 \%$ \\
\hline Tumor maligno de glándulas salivales & C07.X; C08.X & 251 & $3,3 \%$ \\
\hline Melanoma maligno de la piel de cabeza y cuello** & C43.X & 202 & $2,7 \%$ \\
\hline Tumor maligno de fosa nasal y cavidades paranasales & C30.X; C31.X & 167 & $2,2 \%$ \\
\hline Tumor maligno de los ganglios linfáticos de C y C & C77.0 & 63 & $0,8 \%$ \\
\hline Tumor maligno de la hipofaringe & C12.X; C13.X & 61 & $0,8 \%$ \\
\hline Tumor maligno de la nasofaringe & C11.X & 54 & $0,7 \%$ \\
\hline Tumor maligno de la faringe no especificada & C14.2 & 23 & $0,3 \%$ \\
\hline Total & & 7.610 & $100 \%$ \\
\hline
\end{tabular}

El 15,2\% ( $\mathrm{n}=9.887)$ de los egresos fueron por patologías neoplásicas, donde el $77 \%$ correspondió a patología maligna, $9 \%$ a benigna y un $14 \%$ estuvo codificada como de "comportamiento incierto". La mayoría de estos pacientes egresó de instituciones públicas $(53,2 \%)$, un $66,0 \%$ fue sometido a intervención quirúrgica y la mediana de días de hospitalización fue de 2 (R.I. = 1-4). El 61\% correspondió al sexo femenino observando un predominio de población adulta y adulta mayor con un 67,6 y $30,3 \%$ respectivamente. La mediana de edad fue de 55 años (Tablas 4 y 5 ).

Las neoplasias malignas de mayor frecuencia fueron el tumor maligno de tiroides con un $58,3 \%$ de los egresos, tumores malignos localizados en piel $(14,8 \%)$, cavidad oral $(6,9 \%)$, laringe $(5,3 \%)$ y orofaringe $(3,9 \%)$ (Tabla 7$)$. Dentro de los tumores benignos destacan los de glándulas salivales (D11.X) con un 3,4\% del total de las neoplasias (Tabla 7). La Región Metropolitana presentó la mayor proporción 
de egresos por patología neoplásica maligna y al expresarlos en tasa, la Región del Bío-Bío emerge como la región con mayor egreso (Tabla 6).

La patología de oído representó el 7,8\% de los egresos $(n=5.032)$ y, al igual que en las patologías neoplásicas, la mayoría de los egresos fueron del sector público $(53,7 \%)$ con una mediana de días de hospitalización de $1($ R.I $=1-2)$ y resolución quirúrgica en un $76,1 \%$ de los pacientes. No se observaron diferencias por sexo con un predominio de población infantil y adulta, describiendo una mediana de 18 años (R.I = 7-47). El diagnóstico más frecuente fue otitis media no especificada $(29,8 \%)$, seguido por otitis media por efusión (OME) (9,6\%), enfermedades de oído externo $(9,5 \%)$, colesteatoma de oído medio $(8,7 \%)$, otitis media aguda más complicaciones $(5,4 \%)$, "otras otitis medias crónicas" (5,2\%) que corresponden a diagnósticos de otitis media crónica (OMC) sin especificar. Luego se describe oreja alada, otoesclerosis y perforación de la membrana timpánica (Tabla 8).

\begin{tabular}{|c|c|c|c|}
\hline & CIE-10 & $\mathbf{n}$ & $\%$ \\
\hline \multicolumn{4}{|l|}{ Oído } \\
\hline Otitis media no especificada & H66.9 & 1.501 & $29,8 \%$ \\
\hline Otitis media por efusión & $\mathrm{H} 65.2 ; \mathrm{H} 65.3$ & 485 & $9,6 \%$ \\
\hline Enfermedades de oído externo & H60.X; H61.X; H62.X & 476 & $9,5 \%$ \\
\hline Colesteatoma del oído medio & H71.X & 438 & $8,7 \%$ \\
\hline Otitis media aguda y complicaciones & H65.0; H65.1; H66.0; H70.O & 270 & $5,4 \%$ \\
\hline "Otras" otitis medias crónicas & H65.4; H66.1; H66.2; H66.3 & 263 & $5,2 \%$ \\
\hline Oreja alada & Q17.5 & 203 & $4 \%$ \\
\hline Otoesclerosis & H80.X & 196 & $3,9 \%$ \\
\hline Perforación timpánica & H72.X & 195 & $3,9 \%$ \\
\hline Otros & & 1.268 & $20 \%$ \\
\hline Total & & 5.032 & $100 \%$ \\
\hline \multicolumn{4}{|l|}{ Cocleovestibular } \\
\hline Vértigo periférico & $\mathrm{H} 81.0 ; \mathrm{H} 81.1 ; \mathrm{H} 81.2 ; \mathrm{H} 81.3$ & 774 & $45,4 \%$ \\
\hline Vértigo no especificado & H81.8; H81.9; H82.X & 491 & $28,8 \%$ \\
\hline Hipoacusia neurosensorial & H90.3; H90.4; H9O.5 & 128 & $7,5 \%$ \\
\hline Vértigo central & $\mathrm{H} 81.4$ & 103 & $6 \%$ \\
\hline Hipoacusia no especificada & H91.8; H91.9 & 95 & $5,6 \%$ \\
\hline Hipoacusia conductiva & $\mathrm{H} 90.2$ & 64 & $3,8 \%$ \\
\hline Otros & & 50 & $2,9 \%$ \\
\hline Total & & 1.705 & $100 \%$ \\
\hline \multicolumn{4}{|l|}{ Otros vía aerodigestiva superior } \\
\hline Absceso periamigdalino & J36.X & 3.137 & $21,5 \%$ \\
\hline Amigdalitis aguda & J03.X & 1.418 & $9,7 \%$ \\
\hline Fisura labiopalatina & Q35.X; Q36.X; Q37.X & 1.327 & $9,1 \%$ \\
\hline Laringitis aguda & J04.0; J05.X & 1.174 & $8,1 \%$ \\
\hline Enfermedades de las cuerdas vocales y laringe (benigna) & J38.X & 910 & $6,2 \%$ \\
\hline Enfermedades de las glándulas salivales (benigna) & $\mathrm{K} 11 . \mathrm{X}$ & 880 & $6 \%$ \\
\hline Rinofaringitis aguda & J00.X & 818 & $5,6 \%$ \\
\hline Celulitis o absceso de piso de boca & $\mathrm{K} 12.2$ & 555 & $3,8 \%$ \\
\hline Anquiloglosia & Q38.1 & 368 & $2,5 \%$ \\
\hline Cuerpo extraño en vía aérea & T17X & 254 & $1,7 \%$ \\
\hline Otros & & 3.736 & $25,6 \%$ \\
\hline Total & & 14.577 & $100 \%$ \\
\hline
\end{tabular}


La Región Metropolitana mostró el mayor número de egresos por patología de oído con un $49,6 \%$ del total. Sin embargo, la mayor tasa de egreso corresponde a la Región de Aysén (Tabla 6). La patología cócleo-vestibular representó el 2,6\% del total $(\mathrm{n}=1.705)$ con el $52 \%$ de egresos del sector público y un $14,5 \%$ de intervenciones quirúrgicas. Se observó un $55 \%$ de mujeres y una mediana de edad de 55 años $($ R.I. $=36-77)$ con un predominio de adultos y adultos mayores. (Tablas 4 y 5). El diagnóstico con mayor frecuencia fue vértigo periférico $(45,4 \%)$, seguido por vértigo no especificado $(28,8 \%)$, hipoacusia sensorio-neural $(7,5 \%)$, vértigo central $(6,0 \%)$ e hipoacusia no especificada $(5,6 \%)$, dentro de los principales (Tabla 8).

El grupo de patologías benignas de vía aerodigestiva superior representaron el $22,5 \%$ del total nacional $(\mathrm{n}=14.577)$ con un predominio de población infantil (Tabla 5). Dentro de este grupo destacaron el absceso periamigdalino con un $21,5 \%$, enfermedades infecciosas agudas en población pediátrica como amigdalitis aguda $(9,7 \%)$, laringitis aguda $(8,1 \%)$ y rinofaringitis aguda $(5,6 \%)$. Destacan las enfermedades benignas de cuerdas vocales y laringe $(6,2 \%)$ que incluye parálisis cordal, nódulos y pólipos vocales. También, dentro de los más frecuentes se encontró la patología benigna de glándulas salivales $(6,0 \%)$ que incluyó entidades como sialolitiasis y sialoadenitis. Por último, destacar al cuerpo extraño en vía aérea $(1,7 \%)$, el cual es la principal causa de mortalidad en otorrinolaringología ${ }^{3}$ (Tabla 8).

\section{Discusión}

Al observar las características demográficas se aprecia una disminución de la fracción infantil con respecto al estudio de egresos de 1985 , de un $65,0 \%$ a $40,9 \%$ lo cual es concordante con datos demográficos nacionales e internacionales que muestran un envejecimiento de la población ${ }^{5-12}$. Hay que destacar que el trabajo realizado con datos de 1985 tiene criterios de inclusión similares al nuestro por lo cual son comparables. Si bien existe una disminución de la enfermedad crónica de amígdalas (38,0\% en 1985 ; vs. $28,4 \%$ en 2018 ), persiste como la patología más frecuente, concordante con lo descrito en estudios de centros públicos y privados (H.G.B. Concepción 44\%; HJJA: $29,3 \%)^{6,7}$.

La patología rinosinusal y, en particular, la septodesviación, emerge como la segunda enfermedad más frecuente, desplazando a la patología crónica de oído. Esto puede ser explicado por cambios socioeconómicos y aparición de cirugías o resolución de patologías con propósito de mejorar "calidad de vida", fenómeno observado en centros secundarios, tanto en egresos quirúrgicos como de consulta ambulatoria $^{6-11}$. En la misma línea la cirugía del tabique nasal se ve incrementada secundario a un aumento de la cirugía estética nasal, ya que el código FONASA no hace diferencia entre septoplastia y/o rinoplastia.

El aumento en los egresos por enfermedades de origen neoplásico, particularmente cáncer de tiroides, piel, boca y laringe, es consistente con el aumento de las neoplasias a nivel nacional y sugiere ser efecto secundario al envejecimiento de la población y la transición epidemiológica existente en los últimos 20 años $^{12,13}$. Hay que tomar en cuenta que algunos diagnósticos descritos en este estudio corresponden en su mayoría a egresos realizados por otras especialidades como es el caso de laringitis aguda y cáncer de tiroides, resueltos por pediatría y cirugía de cabeza y cuello respectivamente.

$\mathrm{Al}$ estimar la tasa de egreso general por 100.000 habitantes destaca en primer y segundo lugar, la Región de Aysén y Magallanes respectivamente, describiendo las proporciones más altas en enfermedades de oído, amígdalas y patología rinosinusal. Es difícil estimar que esto fuese secundario a una mayor incidencia de enfermedades otorrinolaringológicas y en parte puede ser explicado por una mayor disponibilidad de camas hospitalarias y horas institucionales de especialista para resolución de patologías a nivel secundario, tomando en cuenta si, que esto solo explicaría los egresos públicos y no la tasa global (público más privado) que se describe en este estudio. El año 2018 según estadísticas del MINSAL la Región de Magallanes presentó la mayor cantidad de camas públicas por población beneficiaria de FONASA y en tercer lugar estaría la Región de Aysén con 3,3 y 2,8 camas por 1.000 habitantes respectivamente. En el mismo sentido 
estas regiones describen una buena relación de horas hospitalarias versus población afiliada a FONASA con 11 h/23.832 en la Región de Aysén y 11 h/37.908 para la Región de Magallanes, en comparación con otras regiones como la Metropolitana que en promedio alcanzan $11 \mathrm{~h} / 49.000$ afiliados $^{14,15}$. Tampoco existieron programas de resolución de lista de espera en ambas regiones. Esto se consultó directamente a los servicios de salud respectivos (Aysén y Magallanes) y en ambos casos se señaló que durante el año 2017 y 2018 aumentaron su dotación de médicos otorrinolaringólogos.

Si bien el sistema GRD nos brinda una información detallada de los egresos hospitalarios, se detectaron errores de interpretación de datos al momento de realizar la tabulación de los diagnósticos, lo cual, a nuestro parecer, es la principal limitación del presente estudio. Por ejemplo, en patología de oído existen diversas posibilidades diagnósticas para codificar, que no siempre refleja el concepto clínico de otitis media crónica. La otitis media no especificada (H66.9) y los códigos agrupados como "otras OMC” (H65.4; H66.1; H66.2; H66.3) no especifican a que tipo de otitis media pertenecen, pero al cotejarlos con los códigos quirúrgicos asociados se observa que corresponden a cirugías radicales, timpanoplastías y punciones timpánicas. Esto estaría subestimando la proporción de egresos por perforación de la membrana timpánica (H72X), colesteatoma de oído medio (H71X) y OME.

Algo similar ocurre con la patología cócleo-vestibular, donde llama la atención la gran proporción de vértigos periféricos e hipoacusias que requirieron hospitalización suponiendo que podrían ser ingresos ambulatorios para realizar punciones timpánicas con corticoides o cuadros catalogados como síndromes vestibulares agudos con sospecha de accidente cerebrovascular con necesidad de estudio, de todos éstos sólo el $14,5 \%$ se asoció a una conducta quirúrgica. También se observó que los diagnósticos que hacen referencia a hipoacusias mixtas y de conducción estaban asociados a códigos de cirugías como la estapedostomía y timpanoplastía. En cuanto a las hipoacusias sensorioneurales una baja proporción estaba asociada a laberintectomías y cirugías de neurinoma del nervio acústico. Cabe destacar que estos problemas de codifi- cación sólo se observaron en patología de oído y cócleo vestibular, en donde los diagnósticos GRD disponibles no siguen clasificaciones internacionales como la Sociedad de Barany por ejemplo, facilitando el registro. En los otros grupos diagnósticos también se revisaron los egresos que tenían una conducta quirúrgica sin observar incongruencias similares.

Se debe tener en cuenta que estos datos GRD son codificados por estadísticos no clínicos lo que puede llevar a errores de codificación y registro. Además, es necesario agregar que, en los programas de codificación de diagnósticos habilitados en pabellón y policlínicos, no siempre están disponibles los códigos diagnósticos adecuados, lo cual dificulta un correcto registro. Se podrían generar consensos en codificación de diagnósticos en nuestra especialidad y mayor compromiso con el sistema GRD, para así generar datos más confiables y representativos en el futuro, ya que por el momento ésta es la única fuente de información disponible a nivel nacional.

\section{Conclusión}

El presente estudio es un gran aporte como herramienta de gestión, al dar a conocer la envergadura de los egresos por nuestra especialidad y diagnósticos más frecuentes. Este instrumento debe ser mejorado en futuras revisiones, ampliando los años de estudio y corrigiendo los errores de registro detectados.

\section{Bibliografía}

1. Díaz L, Dávila J, Rodríguez V, Añaños D.

Frecuencia de diagnósticos de la especialidad de otorrinolaringología en el consultorio de medicina general en un centro de salud. Rev Med Hered. 2003; 14(4):163-166.

2. Benito J, Morai D, Miyar V. Estudio descriptivo de la asistencia primaria en otorrinolaringologia. Acta Otorrinolaringol Esp. 1996;47(1):55-62.

3. Béjar M, Cevo J, Romero M, Iñiguez R. Mortalidad nacional en otorrinolaringología. Rev Otorrinolaringol Cir Cabeza Cuello. 2007;67:31-37.

4. Egresos hospitalarios SSMO 2011. Informe preparado por Departamento de Estadística y Gestión de la información. Disponible en: https://degi.saludoriente. cl/degidssmo/info_disp/biodemografica/morbilidad/ 
INFORME_EGRESOS_HOSPITALARIOS_2011.pdf. (Acceso el 2 de septiembre de 2019).

5. Kirschbaum A, Aracena M. Prevalencia de enfermedades de oído, nariz y garganta: Defunciones y egresos en 1985. Rev Otorrinolaringol Cir Cabeza Cuello. 1991;51:9-21.

6. Ulloa P, Urra A, Valenzuela A. Análisis de la atención intrahospitalaria del Servicio de Otorrinolaringología, Hospital Guillermo Grant Benavente de Concepción, 1993-1997. Rev Otorrinolaringol Cir Cabeza Cuello. 1999;59:93-100.

7. Domínguez M, Águila A, Cabrera N, Nazar R, Alzérreca E. Estudio epidemiológico descriptivo de pacientes hospitalizados en el Servicio de ORL del Hospital Clínico de la Universidad de Chile entre los años 2007 y 2014. Rev Otorrinolaringol Cir Cabeza Cuello. 2016;76:265-271.

8. Zapata M. Importancia del sistema GRD para alcanzar la eficiencia hospitalaria. Rev Med Clin Condes. 2018;29(3):347-352.

9. Departamento de Estadísticas e Información en Salud. Egresos Hospitalarios. Disponible en: http:// www.deis.cl/estadisticas-egresoshospitalarios/ (Acceso el 2 de septiembre de 2019).

10. Instituto Nacional de Estadísticas de Chile. Censo 2017. Disponible en: https://www.censo2017.cl/ (Acceso el 18 de noviembre de 2019).

11. Ruz S, Breinbauer H, Arancibia M. Análisis epidemiológico de la patología otorrinolaringológica ambulatoria en el Hospital San Juan de Dios. Rev Otorrinolaringol Cir Cabeza Cuello. 2009;69:227-32.

12. Crespo CF. Chile: nuevos desafíos sanitarios e institucionales en un país en transición. Rev Panam Salud Publica. 2018;42:e137. doi: 10.26633/ RPSP.2018.137.

13. Itriago L, Silva N, Cortes G. Cáncer en Chile y El mundo: Una mirada epidemiológica, presente y futuro. Rev Med Clin Condes. 2013;24(4):351-552.

14. Infraestructura Hospitalaria 2018. Minsal. Disponible en: http://vc.cl/hospitalaria/wp-content/ uploads/2019/04/8h/d1/infraestructura_hospitalaria. pdf.

15. Cardemil F, Barría T, Rahal M, Rodríguez R. Cantidad y distribución de otorrinolaringólogos en los sectores público y privado de salud en Chile. Rev Otorrinolaringol Cir Cabeza Cuello. 2013;73:25-32. 